\title{
Protein kinase CK2 activates Nrf2 via autophagic degradation of Keap1 and activation of AMPK in human cancer cells
}

\author{
Da Eun Jang ${ }^{1, \#}$, Junbin Song ${ }^{1, \#}$, Jeong-Woo Park ${ }^{1}$, Soo-Hyun Yoon ${ }^{2}$ E Young-Seuk Bae ${ }^{1, *}$ \\ ${ }^{1}$ School of Life Sciences, BK21 Plus KNU Creative BioResearch Group, Kyungpook National University, Daegu 41566, ${ }^{2}$ Research Institute \\ of Pharmaceutical Sciences, College of Pharmacy, Kyungpook National University, Daegu 41566, Korea
}

\begin{abstract}
Protein kinase CK2 downregulation induces premature senescence in various human cell types via activation of the reactive oxygen species (ROS)-p53-p21 ${ }^{\text {Cip1/WAF1 }}$ pathway. The transcription factor "nuclear factor erythroid 2-related factor 2" (Nrf2) plays an important role in maintaining intracellular redox homeostasis. In this study, Nrf2 overexpression attenuated CK2 downregulationinduced ROS production and senescence markers including SA- $\beta$-gal staining and activation of p53-p21 ${ }^{\text {Cip1/WAF1 }}$ in human breast (MCF-7) and colon (HCT116) cancer cells. CK2 downregulation reduced the transcription of Nrf2 target genes, such as glutathione S-transferase, glutathione peroxidase 2, and glutathione reductase 1. Furthermore, CK2 downregulation destabilized Nrf2 protein via inhibiting autophagic degradation of Kelch-like ECHassociated protein 1 (Keap1). Finally, CK2 downregulation decreased the nuclear import of Nrf2 by deactivating AMP-activated protein kinase (AMPK). Collectively, our data suggest that both Keap1 stabilization and AMPK inactivation are associated with decreased activity of Nrf2 in CK2 downregulation-induced cellular senescence. [BMB Reports 2020; 53(5): 272-277]
\end{abstract}

\section{INTRODUCTION}

Cellular senescence has been defined as a classic hallmark of the aging process. It can be triggered by various forms of stress, including telomere shortening, oncogenic activation, and reactive oxygen species (ROS), which induce DNA damage $(1,2)$. DNA damages result in the stabilization of p53 and subsequent overexpression of $\mathrm{p} 21^{\mathrm{Cip} 1 / \mathrm{WAF} 1}$, which then inhibits cell cycle progression. Therefore, it has been assumed that cellular senescence is a beneficial compensatory response that prevents the propagation of damaged and potentially tumorigenic cells $(1,2)$.

${ }^{*}$ Corresponding author. Tel: +82-53-950-6355; Fax: +82-53-9432762; E-mail: ysbae@knu.ac.kr

${ }^{\#}$ These authors contributed equally to this work.

https://doi.org/10.5483/BMBRep.2020.53.5.044

Received 25 February 2020, Revised 27 March 2020, Accepted 31 March 2020

Keywords: AMPK, Antioxidants, CK2, Keap1, Nrf2
Senescent cells can be identified by several molecular and cytological features, including retinoblastoma protein (RB) hypophosphorylation, SA- $\beta$-gal activity, the formation of senescence-associated heterochromatin foci, secretion of several inflammatory cytokines and chemokines, and upregulation of cell cycle arrest genes, such as p16 ${ }^{\mathrm{INK} 4 \mathrm{~A}}, \mathrm{ARF}, \mathrm{p} 53$ and p21 $1^{\text {Cip1/NAF1 }}(3-5)$.

Previously, it has been reported that CK2 activity is downregulated in senescent IMR-90 human lung fibroblast cells, aged rat tissues, and aged nematodes $(6,7)$. Further, CK2 downregulation induces premature senescence in various human cells via activation of the ROS-p53-p2 $1^{\text {Cip1/NAF1 }}$ pathway $(8,9)$. Intracellular ROS homeostasis is maintained by the balance between the mechanisms that generate ROS and those that neutralize such toxic oxidants. The ROS detoxifying factors include $\mathrm{Cu} / \mathrm{Zn}$ superoxide dismutase (SOD), MnSOD, catalase, glutathione S-transferase (GST), glutathione peroxidase 2 (GPX2), and glutathione reductase 1 (GSR1) $(10,11)$. We have shown that CK2 downregulation stimulates ROS accumulation via AKT-mediated inhibition of the antioxidant transcription factor FoxO3a. AKT-mediated phosphorylation of FoxO3a results in nuclear export of FoxO3a and thereby decreases transcription of FoxO3a target genes, such as $\mathrm{Cu} / \mathrm{ZnSOD}, \mathrm{MnSOD}$, and catalase (12). The transcription factor $\mathrm{Nrf} 2$ also participates in the maintenance of redox homeostasis in the cell. Nrf2 transcriptional response is mediated through the antioxidant response element (ARE) at the enhancer regions of its target genes, such as GST, GPX2, and GSR1 $(13,14)$. Nrf2 activity can be controlled by post-translational modifications. The best characterized mechanism for the regulation of Nrf2 activity is at the level of protein stability by Keap1, which is an adaptor protein for a Cul3based ubiquitin E3 ligase. Under physiological conditions, Keap1 binds to and promotes the ubiquitination of Nrf2 in the cytoplasm, resulting in the proteasomal degradation of Nrf2. Under oxidative conditions, the ubiquitin E3 ligase activity of the Keap1-Cul3 complex declines. Consequently, Nrf2 is stabilized and translocates into the nucleus $(14,15)$.

In this study, we examined the role of Nrf2 in the ROS generation during CK2 downregulation-induced senescence. Our results suggest that $\mathrm{CK} 2$ downregulation inhibits the nuclear import of Nrf2 through decreasing AMPK activity and increasing Keap1 stability, consequently increasing ROS levels in cells.

ISSN: 1976-670X (electronic edition)

Copyright (C) 2020 by the The Korean Society for Biochemistry and Molecular Biology

(c) This is an open-access article distributed under the terms of the Creative Commons Attribution Non-Commercial License (http://creativecommons.org/licenses/by-nc/4.0) which permits unrestricted non-commercial use, distribution, and reproduction in any medium, provided the original work is properly cited. 


\section{RESULTS}

\section{Overexpression of Nrf2 attenuates CK2 downregulation-induced upregulation of senescence markers and production of ROS in human cancer cells}

To investigate the involvement of Nrf2 in CK2 downregulationinduced senescence, MCF-7 and HCT116 cells were transfected with CK2 $\alpha$ siRNA along with Nrf2 cDNA. As noted in previous reports $(6,8,9)$, CK2 downregulation upregulated senescence markers, such as p53 and p21 Cip1/WAF1 levels and SA- $\beta$-gal activity. However, this effect was successfully suppressed in CK2-downregulated cells upon Nrf2 overexpression (Fig. 1A and 1B). We then analyzed whether Nrf2 was involved in CK2 downregulation-induced ROS production. Knocking down CK2 $\alpha$ increased ROS production as evaluated by $\mathrm{CM}-\mathrm{H}_{2}$ DCFDA staining. However, overexpression of $\mathrm{Nrf} 2$ antagonized the increase in ROS levels induced by CK2 downregulation (Fig. 1C). Taken together, these results suggest that downregulation of $\mathrm{Nrf2}$ is required for CK2 downregulation-induced ROS production and senescent phenotypes.
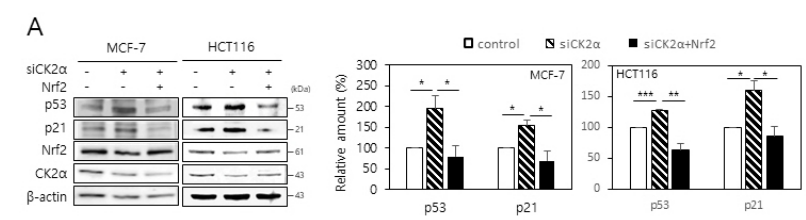

B
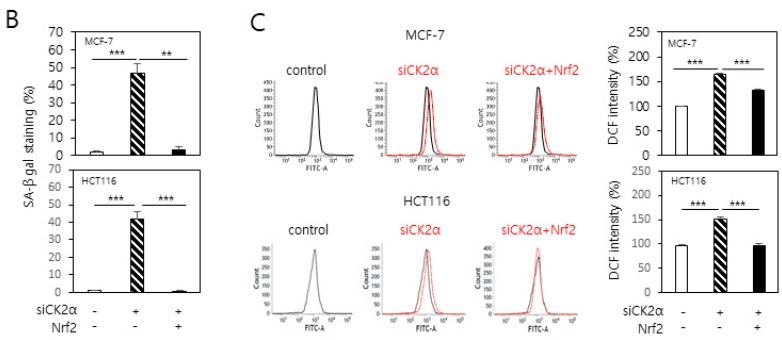

Fig. 1. Overexpression of Nrf2 attenuates the CK2 downregulationinduced ROS production and senescence phenotypes in human cancer cells. MCF-7 and HCT116 cells were transfected with CK2 $\alpha$ siRNA in the absence or presence of the Nrf2 cDNA for $48 \mathrm{~h}$. (A) Cells were lysed and electrophoresed on a 10\% SDS-polyacrylamide gel. Protein bands were visualized by immunoblotting (left panel). Representative data from three independent experiments are shown. Graphs show the quantification of the protein levels relative to that of $\beta$ actin (right panel). (B) Cells were stained with 5-bromo-4-chloro-3-indolyl$\beta$-D-galactoside, and the percentage of blue-stained cells was measured.

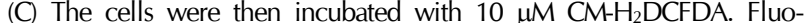
rescence intensity was determined by flow cytometry analysis (left panel). Representative data from three independent experiments are shown. The graphs show the relative fluorescence level (right panel). All data are presented as means \pm SEM. ${ }^{* P}<0.05 ; * *<0.01$; $* * * P<0.001$.

\section{CK2 downregulation reduces Nrf2 protein level in human cancer cells}

To determine the role of CK2 in the regulation of Nrf2 activity, MCF-7 and HCT116 cells were transfected with an ARE-luciferase construct along with either the CK2 $\alpha$ siRNA or pcDNAHA-CK2 $\alpha$. CK2 downregulation decreased the activity of the luciferase reporter by approximately 30-50\% compared with the control level. Conversely, the overexpression of $\mathrm{CK} 2 \alpha$ increased the luciferase activity by approximately $20 \%$ (Fig. 2A). It has been reported that $\mathrm{Nrf} 2$ regulates the expression of several antioxidant genes, such as GST, GPX2, and GSR1 (13, 14). Our reverse transcription (RT)-PCR analysis also demonstrated that Nrf2 overexpression increased the GST, GPX2, and GSR1 mRNA levels in the cells (Supplementary Fig. 1). To examine the effects of CK2 on the transcription of these Nrf2 target genes, cells were transfected with the CK2 $\alpha$ siRNA or pcDNAHA-CK2 $\alpha$. Whereas CK2 $\alpha$ knockdown decreased the GST, GPX2, and GSR 1 mRNA levels, CK2 $\alpha$ overexpression increased these mRNA levels (Fig. 2B). Next, we examined whether CK2 regulated the expression of Nrf2. Immuno-blot analysis indicated that $\mathrm{CK} 2 \alpha$ downregulation decreased the Nrf2 protein level in the cells. Conversely, the overexpression of CK2 $\alpha$ increased the Nrf2 protein level (Fig. 2C). Taken together, these results suggest that CK2 downregulation reduces Nrf2 protein levels, and consequently, the transcription of Nrf2 target genes decrease

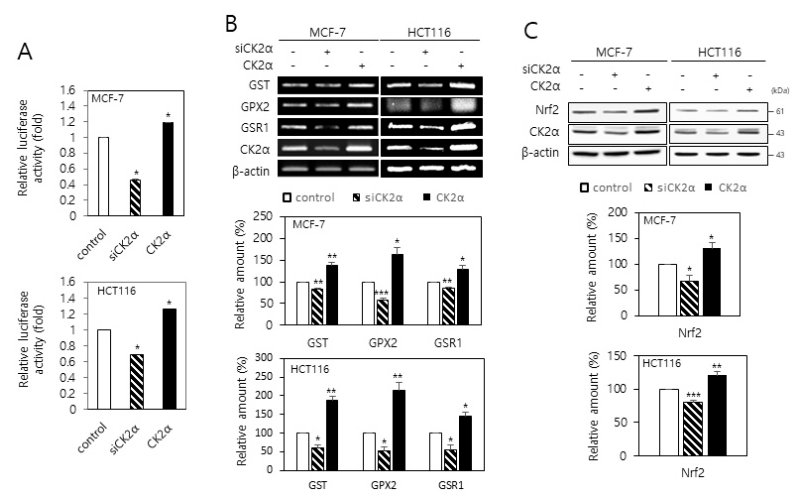

Fig. 2. CK2 downregulation reduces the transcriptional activity and expression of Nrf2 in human cancer cells. (A) MCF-7 and HCT116 cells were co-transfected with the ARE luciferase construct and CK2 $\alpha$ siRNA or pcDNA-HA-CK $2 \alpha$. The firefly luciferase activity was measured $24 \mathrm{~h}$ after transfection and normalized to Renilla luciferase activity. (B, C) Cells were transfected with CK2 $\alpha$ siRNA or pcDNAHA-CK2 $\alpha$ for 48 h. (B) Total RNA was extracted from the cells, and RT-PCR was performed using specific primers. PCR products were resolved on a 1.5\% agarose gel (upper panel). Graphs show the quantification of the mRNA levels of each gene relative to that of $\beta$-actin (bottom panels). (C) Cells were lysed and electrophoresed on a $10 \%$ SDS-polyacrylamide gel. Protein bands were visualized by immunoblotting (upper panel). Graphs show the quantification of the protein levels relative to $\beta$-actin levels (bottom panels). All data are shown as means \pm SEM. $* P<0.05 ; * * P<$ $0.01 ; * * * P<0.001$. 
in human cancer cells.

\section{CK2 increases autophagic degradation of Keap1 in human cancer cells}

To investigate the mechanism by which CK2 downregulation decreases Nrf2 protein level, CK2-downregulated cells were treated with the proteasome inhibitor MG132 $(10 \mu \mathrm{M})$. The CK2 downregulation-induced decrease in Nrf2 was attenuated by the treatment with MG132, suggesting that CK2 downregulation stimulates proteasomal degradation of Nrf2 (Fig. 3A). Because Keap1 promotes proteasomal degradation of $\mathrm{Nrf} 2$ and thus acts as a negative regulator of $\mathrm{Nrf} 2(14,15)$, we examined whether CK2 regulated Nrf2 protein level via Keap1. As shown in Fig. 3B, CK2 $\alpha$ downregulation increased the Keap1 protein level in the cells, and the upregulation had the opposite effect. We then tested the role of autophagy in CK2-mediated Keap1 downregulation. Treatment with the autophagy inhibitors chloroquine $(C Q, 100 \mu \mathrm{M})$, 3-methyladenine (3-MA, $1 \mathrm{mM})$, or ATG5 siRNA abolished the CK2 overexpression-induced downregulation of Keap1, suggesting that CK2 negatively controls Keap1 protein level through autophagy (Fig. 3C and 3D). Taken together, these data suggest that CK2 protects Nrf2 from proteasomal degradation via stimulating the autophagic degradation of Keap1.

\section{CK2 downregulation reduces the nuclear localization of Nrf2} by inhibiting AMPK in human cancer cells

To examine the involvement of CK2 in the nuclear localization
A
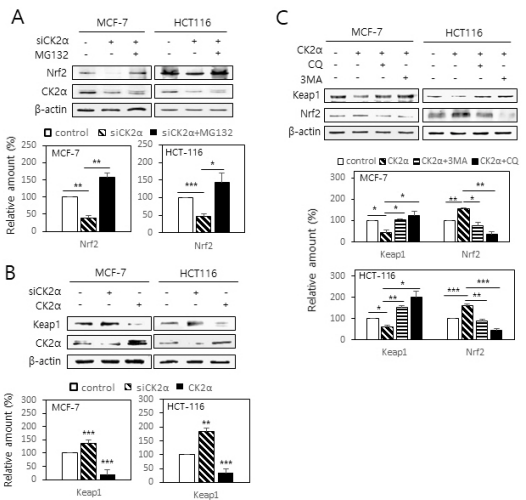
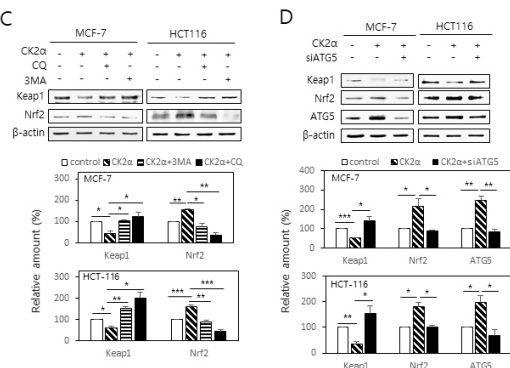

Fig. 3. CK2 downregulation stimulates proteasomal degradation of Nrf2 via increasing Keap1 stability. (A) Cells were transfected with CK2 $\alpha$ siRNA in the presence or absence of the proteasome inhibitor MG132 $(10 \mu \mathrm{M})$. (B) Cells were transfected with CK2 $\alpha$ siRNA or pcDNA-HA-CK2 $\alpha$ for 48 h. (C, D) Cells were transfected with pcDNA-HA-CK $2 \alpha$ in the presence or absence of the autophagy inhibitor chloroquine $(C Q, 100 \mu \mathrm{M})$, 3-methyladenine (3-MA, $1 \mathrm{mM}$ ) (C), or ATG5 siRNA (D). Cells were lysed and electrophoresed on a $10 \%$ SDS-polyacrylamide gel. Protein bands were visualized by immunoblotting (upper panels). Graphs show the quantification of the protein levels relative to $\beta$-actin level (bottom panels). All data are shown as means \pm SEM. $* \mathrm{P}<0.05 ; * * \mathrm{P}<0.01 ; * * * \mathrm{P}<0.001$. of NRF2, we separated cytoplasm and nuclei from the cells transfected with CK2 $\alpha$ siRNA or pcDNA-HA-CK2 $\alpha$. More Nrf2 was found in the nuclear extracts than in the cytosolic extracts of the cells overexpressing CK2 $\alpha$. In contrast, less Nrf2 was observed in the nuclear extracts than in the cytosolic extracts of cells lacking $\mathrm{CK} 2 \alpha$, implying that $\mathrm{CK} 2$ downregulation inhibits the nuclear import of Nrf2 (Fig. 4A). It has been reported that the $\alpha$ subunit of AMPK is phosphorylated at the T172 residue upon activation $(16,17)$, and AMPK acts as an upstream regulator of Nrf2 (18). Thus, we examined the phosphorylation status of this residue by immuno-blot analysis to assess whether CK2 regulated the activity of AMPK. Whereas CK2 downregulation decreased the phosphorylation of the T172 residue of AMPK $\alpha$ subunit, CK2 $\alpha$ overexpression had the opposite effect, indicating that CK2 induces AMPK activity (Fig. 4B). To determine the role of AMPK in ARE-mediated transcription, cells expressing the ARE-luciferase reporter were treated with the AMPK activator AICAR. Treatment with AICAR $(100 \mu \mathrm{M})$ completely abolished CK2 downregulationinduced reduction in the ARE-luciferase activity, suggesting that AMPK acts as a regulator of Nrf2 in senescent cells made by
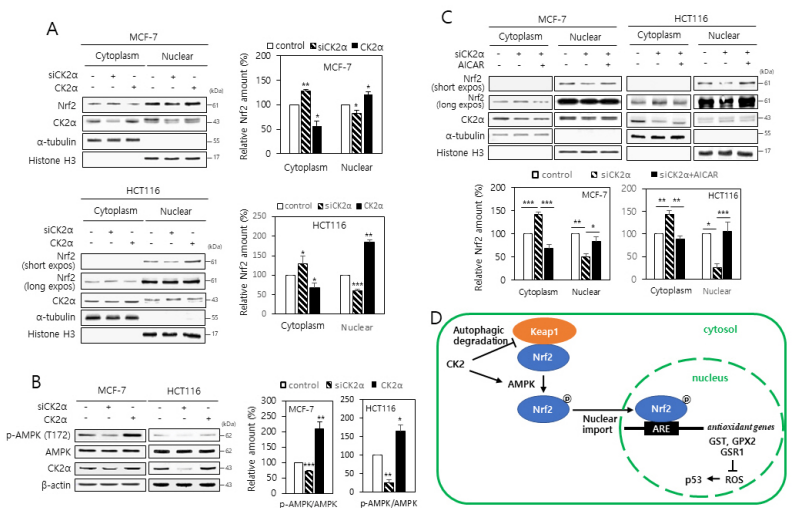

Fig 4. CK2 downregulation reduces the nuclear localization of Nrf2 through inhibiting AMPK in human cancer cells. (A) Cytoplasmic and nuclear fractions were isolated from the cells transfected with CK2 $\alpha$ siRNA or pcDNA3.1-HA-CK2 $\alpha$ and then both extracts were analyzed by immunoblotting (left panels). $\alpha$-Tubulin (cytoplasmic marker) and histone $\mathrm{H} 3$ (nuclear marker) levels were quantified as loading controls. Graphs present the quantification of Nrf2 levels relative to the subcellular marker levels (right panels). (B) Cells were transfected with CK2 $\alpha$ siRNA or pcDNA3.1-HA-CK2 $\alpha$ for $48 \mathrm{~h}$, lysed, and electrophoresed on a 10\% SDS-polyacrylamide gel. Protein bands were visualized by immunoblotting (upper panel). Graphs show the quantification of the p-AMPK levels relative to AMPK level (bottom panel). (C) Cytoplasmic and nuclear extracts were isolated from the cells, and then both extracts were analyzed by immunoblotting (upper panels). The levels of $\alpha$-tubulin (cytoplasmic marker) and histone $\mathrm{H} 3$ (nuclear marker) were quantified as loading controls. Graphs represent the quantification of Nrf2 levels relative to subcellular marker levels (bottom panels). All the data are shown as means + SEM. $* \mathrm{P}<0.05 ; * * \mathrm{P}<0.01 ; * * * \mathrm{P}<$ 0.001 . (D) Mechanistic model illustrating the involvement of Nrf2 in the CK2 downregulation-induced cellular senescence. 
CK2 downregulation (Supplementary Fig. 2). We then investigated the role of AMPK in Nrf2 nuclear export induced by CK2 downregulation. As shown in Fig. 4C, the CK2 downregulationinduced nuclear export of Nrf2 was attenuated by treatment with AICAR. Collectively, these results demonstrate that the AMPK-Nrf2 signaling pathway is suppressed during the cellular senescence induced by CK2 downregulation.

\section{DISCUSSION}

CK2 downregulation has been shown to induce premature senescence in IMR-90 human lung fibroblast cells and in HCT116 and MCF-7 human cancer cells $(6,8,9)$. Additionally, kin-10 (C. elegans ortholog of CK2 $\beta$ ) knockdown induces the expression of age-related biomarkers in C. elegans (7). CK2 downregulation increases ROS accumulation by downregulating FoxO3a and DAF-16 (C. elegans ortholog of FoxO3a) activity in human cells and C. elegans, respectively $(12,7)$, indicating that an increase in ROS levels plays an important role in CK2 downregulation-induced senescence and aging. The present study demonstrates that Nrf2 downregulation is associated with ROS generation during CK2 downregulation-mediated senescence in human cancer cells. In this study, Nrf2 overexpression abrogated the upregulation of CK2 downregulationinduced senescence markers (SA- $\beta$-gal staining and activation of $\mathrm{p} 53-\mathrm{p} 21^{\mathrm{Cip} 1 / \mathrm{WAF} 1}$ ) and ROS production (Fig. 1). In addition, CK2 downregulation decreased the Nrf2 protein level and the mRNA levels of Nrf2 target genes (GST, GPX2, and GSR1) (Fig. 2), suggesting that CK2 positively regulates Nrf2 activity.

The present results show that CK2 regulates Nrf2, at least, by two pathways. First, CK2 regulates the level of Nrf2 protein via Keap1. The present study demonstrated that CK2 downregulationinduced decrease in the $\mathrm{Nrf} 2$ protein was reversed by treatment with the proteasome inhibitor MG132 (Fig. 3A). In addition, CK2 upregulation decreased the level of Keap1 protein, a component of Cullin-3-type ubiquitin ligase for Nrf2, and this event was suppressed by treatments with autophagy inhibitors (chloroquine, 3-methyladenine, or ATG5 siRNA) (Fig. 3B-D). Taken together, these results suggest that $\mathrm{CK} 2$ reduces proteasomal $\mathrm{Nrf} 2$ degradation by stimulating the autophagic degradation of Keap1. Furthermore, reduction in Keap1 levels may allow accumulation of Keap1-free Nrf2 in the cytoplasm and consequently, Nrf2 translocates into the nucleus (Fig. 4D). This conclusion is consistent with the results from previous reports have shown that the autophagy adaptor p62 directly interacts with Keap1, thereby directing Keap1 toward autophagic degradation $(19,20)$. Second, CK2 regulates the nuclear translocation of Nrf2 via AMPK. The present study found that CK2 downregulation inhibited the nuclear import of Nrf2 (Fig. 4A). CK2 downregulation decreased the activity of AMPK, and treatment with the AMPK activator AICAR rescued CK2 downregulation-induced reduction in the Nrf2 activity (Fig. 4B and Supplementary Fig. 2). Further, treatment with AICAR attenuated the CK2 downregulation-induced nuclear export of
Nrf2 (Fig. 4C), indicating that CK2 downregulation inhibits the nuclear import of $\mathrm{Nrf} 2$ by reducing the AMPK activity. Collectively, these results indicates that CK2 downregulation inhibits Nrf2 activity via both stabilization of Keap1 and inactivation of AMPK, consequently decreasing the intracellular antioxidant level, leading to ROS accumulation and cellular senescence (Fig. 4D). We currently cannot explain how CK2 increases AMPK activity and autophagic degradation of Keap1. However, since senescent cells accumulate with age in various tissues and species, and have been observed in various age-related diseases $(3,21-23)$, elucidating the molecular mechanism by which CK2 regulates the Keap1-Nrf2 axis will extend our understanding of organismal aging and contribute to the development of anti-senescence/aging therapies.

\section{MATERIALS AND METHODS}

\section{Cell culture, RNA interference, and DNA transfection}

MCF-7 human breast cancer cells and HCT116 human colon cancer cells (ATCC, Manassas, VA) were cultured in Dulbecco's Modified Eagle Medium containing $10 \%(\mathrm{v} / \mathrm{v})$ fetal bovine serum under a humidified atmosphere of $5 \%(\mathrm{v} / \mathrm{v}) \mathrm{CO}_{2}$ at $37^{\circ} \mathrm{C}$. pcDNA3-EGFP-C4-Nrf2 and pcDNA3.1-HA-CK2 $\alpha$ were transfected into cells using Lipofectamine 2000 (Invitrogen, Carlsbad, CA) as described by the manufacturer's instruction. ATG5siRNA (ATG5 HSS114104) was purchased from Invitrogen. siRNAs were transfected into cells using Polyfect (Invitrogen) for $48 \mathrm{~h}$. The sequence of CK $2 \alpha$ siRNA was 5'-UCAAGAUGACUACCA GCUGdTdT-3', and that of the negative control was 5'-GCUC AGAUCAAUACGGAGAdTdT-3'.

\section{RT-PCR}

Total RNA was extracted from HCT116 and MCF-7 cells and reverse transcribed using reverse transcriptase (Takara Bio Inc., Kyoto, Japan) and gene-specific reverse primers. The resulting cDNAs were PCR-amplified. The primers used for the assays are listed in Supplementary Table 1. PCR products were resolved on a $1.5 \%$ agarose gel. The RT-PCR bands were quantified using densitometry. $\beta$-actin RNA was used as an internal control to standardize the RNA amount in each sample.

\section{Immuno-blotting}

Antibodies against Nrf2, CK2 $\alpha$, p53, p2 $1^{\text {Cip1/NAF1 }}$, Keap1 and $\beta$-actin were obtained from Santa Cruz Biotechnology (Santa Cruz, CA). Antibodies specific for AMPK and phospho-AMPK (T172) were obtained from Cell Signaling Technology (Beverly, MA). Proteins were separated on $10 \%$ polyacrylamide gels in the presence of SDS, and then transferred onto nitrocellulose membranes. The membranes were blocked with $5 \%(\mathrm{w} / \mathrm{v})$ non-fat, dried skim milk in TBST $[20 \mathrm{mM}$ Tris- $\mathrm{HCl}(\mathrm{pH} 7.4)$, $150 \mathrm{mM} \mathrm{NaCl}$, and $0.05 \%$ Tween 20] for $2 \mathrm{~h}$ and then incubated with specific antibodies in $1 \%(\mathrm{w} / \mathrm{v})$ non-fat, dried skim milk for $1 \mathrm{~h}$. The membranes were washed three times with TBST, and then treated with the ECL system for the 
development of the signals (Amersham, GE Healthcare, Little Chalfont, UK). When deemed necessary, membranes were stripped with the stripping buffer [2\% SDS, $100 \mathrm{mM} \beta$-mercaptoethanol, and $50 \mathrm{mM}$ Tris- $\mathrm{HCl}\left(\mathrm{pH} \mathrm{7.0)]}\right.$ at $50^{\circ} \mathrm{C}$ for $1 \mathrm{~h}$ with gentle shaking and then re-probed with anti- $\beta$-actin antibody as an internal loading control.

\section{SA- $\beta$-gal activity assay}

Cells in sub-confluent cultures were washed with ice-cold phosphate-buffered saline (PBS), fixed with $3 \%(\mathrm{v} / \mathrm{v})$ formaldehyde in PBS for $10 \mathrm{~min}$ at room temperature, and then incubated with a staining solution containing $1 \mathrm{mg} / \mathrm{ml}$ 5-bromo-4-chloro3 -indolyl $\beta$-D-galactoside, $40 \mathrm{mM}$ citric acid-sodium phosphate ( $\mathrm{pH}$ 6.0), $5 \mathrm{mM}$ potassium ferricyanide, $5 \mathrm{mM}$ potassium ferrocyanide, $150 \mathrm{mM} \mathrm{NaCl}$, and $2 \mathrm{mM} \mathrm{MgCl}$ for $24 \mathrm{~h}$ at $37^{\circ} \mathrm{C}$. Blue-stained cells were counted in at least 10 fields at $400 \times$ magnification, and expressed as a percentage of positive cells.

\section{Isolation of nuclear and cytoplasmic extract}

Cytoplasmic and nuclear extracts were prepared using the NE-PER Nuclear and Cytoplasmic Extraction Reagent Kit (Pierce, Rockford, IL) according to the manufacturer's instruction.

\section{Dual-luciferase reporter assay}

For the measurement of ARE activity, $1 \times 10^{4}$ cells were transfected along with an ARE reporter vector (Promega, Korea) and phRL-TK Renilla luciferase vector (Promega) or inhibitor in a 96-well plate using Lipofectamine 2000 according to the manufacturer's protocol. After $24 \mathrm{~h}$, firefly and Renilla luciferase activities were consecutively measured using the Dual-Luciferase Reporter Assay System (Promega).

\section{Measurement of intracellular ROS levels}

The oxidation-sensitive fluorescent probe $\mathrm{CM}_{2} \mathrm{H}_{2}$ DCFDA (Invitrogen) was used to evaluate ROS levels. Cells were treated with $5 \mu \mathrm{M}$ $\mathrm{CM}-\mathrm{H}_{2}$ DCFDA for $20 \mathrm{~min}$ at $37^{\circ} \mathrm{C}$ in the dark, detached by trypsinization, and then washed with PBS. Fluorescence intensity was determined with a Coulter Elite ESP Cell Sorter (Beckman Coulter Inc., Brea, CA). The forward and side scatter gates were set to exclude any dead cells from the analysis; at least 10,000 events within the gate were acquired per sample.

\section{Statistical Analysis}

One-way analysis of variance testing of the data was performed using the SPSS package program (IBM, Armonk, NY). The results were considered significant if the $\mathrm{P}$ value was $<$ 0.05. Duncan's multiple-range test was performed if the differences between the groups were identified as $\alpha=0.05$.

\section{ACKNOWLEDGEMENTS}

This research was supported by the Basic Science Research Program through the National Research Foundation of Korea (NRF) funded by the Ministry of Science, ICT and Future Plan- ning (NRF-2019R1A2C1005219).

\section{CONFLICTS OF INTEREST}

The authors have no conflicting interests.

\section{REFERENCES}

1. Campisi J (2013) Aging, cellular senescence, and cancer. Annu Rev Physiol 75, 685-705

2. Kuilman T, Michaloglou C, Mooi WJ et al (2010) The essence of senescence. Genes Dev 24, 2463-2479

3. Dimri GP, Lee X, Basile G et al (1995) A biomarker that identifies senescent human cells in culture and in aging skin in vivo. Proc Natl Acad Sci U S A 92, 9363-9367

4. Coppé JP, Desprez PY, Krtolica A et al (2010) The senescence-associated secretory phenotype: the dark side of tumor suppression. Annu Rev Pathol 5, 99-118

5. Narita M, Nunez S, Heard E et al (2003) Rb-mediated heterochromatin formation and silencing of E2F target genes during cellular senescence. Cell 113, 703-716

6. Ryu SW, Woo JH, Kim YH et al (2006) Downregulation of protein kinase CKII is associated with cellular senescence. FEBS Lett 580, 988-994

7. Park JH, Lee JH, Park JW et al (2017) Downregulation of protein kinase CK2 activity induces age-related biomarkers in C. elegans. Oncotarget 8, 36950-36963

8. Kang JY, Kim JJ, Jang SY et al (2009) The p53-p21 Cip1/WAF1 pathway is necessary for cellular senescence induced by the inhibition of protein kinase CKII in human colon cancer cells. Mol Cells 28, 489-494

9. Jeon SM, Lee SJ, Kwon TK et al (2010) NADPH oxidase is involved in protein kinase CKII down-regulation-mediated senescence through elevation of the level of reactive oxygen species in human colon cancer cells. FEBS Lett $584,3137-3142$

10. Liou GY and Storz P (2010) Reactive oxygen species in cancer, Free Radic Res 44, 479-496

11. Bokov A, Chaudhuri A and Richardson A (2004) The role of oxidative damage and stress in aging. Mech Ageing Dev 125, 811-826

12. Park SY and Bae YS (2016) Inactivation of the FoxO3a transcription factor is associated with the production of reactive oxygen species during protein kinase CK2 downregulation-mediated senescence in human colon cancer and breast cancer cells. Biochem Biophys Res Commun $478,18-24$

13. Tebay LE, Robertson H, Durant T et al (2015) Mechanisms of activation of the transcription factor Nrf2 by redox stressors, nutrient cues, and energy status and the pathways through which it attenuates degenerative disease. Free Radic Biol Med 88, 108-146

14. Hayes JD, McMahon M, Chowdhry S et al (2010) Cancer chemoprevention mechanisms mediated through the Keap1Nif2 pathway. Antioxid Redox Signal 13, 1713-1748

15. Itoh K, Mimura J and Yamamoto M (2010) Discovery of the negative regulator of Nrf2, Keap1: a historical overview. Antioxidants Redox Signal 13, 1665-1678

16. Hardie DG, Ross FA and Hawley SA (2012) AMPK: a 
nutrient and energy sensor that maintains energy homeostasis. Nat Rev Mol Cell Biol 13, 251-262

17. Salminen A and Kaarniranta K (2012) AMP-activated protein kinase (AMPK) controls the aging process via an integrated signaling network. Ageing Res Rev 11, 230-241

18. Joo MS, Kim WD, Lee KY et al (2016) AMPK facilitates nuclear accumulation of Nrf2 by phosphorylating at serine 550. Mol Cell Biol 36, 1931-1942

19. Komatsu M, Kurokawa $H$, Waguri $S$ et al (2010) The selective autophagy substrate p62 activates the stress responsive transcription factor $\mathrm{Nrf} 2$ through inactivation of Keap1. Nat Cell Biol 12, 213-223

20. Lau A, Wang XJ, Zhao F et al (2010) A noncanonical mechanism of Nrf2 activation by autophagy deficiency: direct interaction between Keap1 and p62. Mol Cell Biol 30, 3275-3285

21. Herbig U, Ferreira $M$, Condel $L$ et al (2006) Cellular senescence in aging primates. Science (New York, NY) 311,1257

22. Jeyapalan JC, Ferreira M, Sedivy JM et al (2007) Accumulation of senescent cells in mitotic tissue of aging primates. Mech Ageing Dev 128, 36-44

23. Kwon SM, Hong SM, Lee YK et al (2019) Metabolic features and regulation in cell senescence. BMB Rep 52, 5-12 